\title{
Genetic Variant of the C-reactive Protein Gene and Prevalence of Peripheral Arterial Disease in Patients with Type 2 Diabetes Mellitus
}

Stavroula Papaoikonomou ${ }^{1 * \#}$, Dimitris Tousoulis ${ }^{2 * \#}$, Nicholas Tentolouris $^{1}$, Nikolaos Papageorgiou ${ }^{2}$, Antigoni Miliou ${ }^{2}$, Emmanuel Androulakis $^{2}$, Charalambos Antoniades ${ }^{2}$ and Christodoulos Stefanadis ${ }^{2}$

${ }^{1}$ Department of Propaedeutic and Internal Medicine, Athens University Medical School, Laiko General Hospital, Greece

${ }^{2}$ Cardiology Department, Athens University Medical School, Hippokration General Hospital, Greece

\#Authors contributed equally

\begin{abstract}
Objective: Diabetes mellitus is strongly associated with inflammatory procedures, obesity and macrovascular complications, while genetic factors are involved in the disease pathogenesis and its complications. The aim of this study was to examine the impact of $A 3872 \mathrm{G}$ polymorphism on C-reactive protein (CRP) gene on the prevalence of peripheral arterial disease (PAD) in Greek Caucasian subjects with type 2 diabetes mellitus (T2DM)

Methods: The study population consisted of 423 patients with T2DM. The A3872G polymorphism was detected by polymerase chain reaction and appropriate restriction enzyme digestion $(\mathrm{HpyCH} 4 \mathrm{III})$. High sensitivity CRP was assayed by particle-enhanced immunonephelometry. PAD was diagnosed using clinical or ultrasonography criteria and/or ankle brachial index $<0.9$

Results: The genotype distribution (\%) in PAD patients (GG: 48.1, AG: 29.1, AA: 22.8) did not differ significantly compared to non-PAD disease subjects (GG: 53.8, AG: 26.7, AA: 19.5), ( $P=0.258)$. After adjustment for classical risk factors, carriers of " $A$ " allele (AG+AA) compared with GG homozygotes had higher odds (OR) for PAD, (AG+AA): 82 (40.0) vs. GG: 76 (34.2), (OR 1.622, 95\% confidence intervals 1.029-2.536, $P=0.037$ ).

Conclusions: In Caucasian subjects with T2DM, the GG homozygotes of the A3872G genetic polymorphism on the $\mathrm{C}$-reactive protein gene may be protected from the risk of peripheral arterial disease compared to carriers of the "A" allele. These results indicate a potential genetic role of inflammation in atherosclerosis in patients with type 2 diabetes mellitus.
\end{abstract}

Keywords: Type 2 diabetes mellitus; Peripheral arterial disease; C-reactive protein; Gene polymorphism; Waist-to-hip ratio

\begin{abstract}
Abbreviations: CRP: C-reactive Protein; T2DM: Type 2 Diabetes Mellitus; PAD: Peripheral Arterial Disease; WHR: Waist-to-hip Ratio; CVD: Cardiovascular Disease; ABI: Ankle Brachial Pressure Index; BMI: Body Mass Index; NCEP: National Cholesterol Education Program; PCR: Polymerase Chain Reaction; OR: Odds Ratio; CI: Confidence Intervals; HbA1c: Glycated Hemoglobin; GFR: Glomerular Filtration Rate; HDL-C: High-density Lipoprotein Cholesterol; LDL-C: Low-density Lipoprotein Cholesterol
\end{abstract}

\section{Introduction}

The high prevalence of type 2 diabetes mellitus (T2DM) in patients with vascular disease is well established $[1,2]$ and it accounts for the majority of risk associated with development of clinically significant peripheral arterial disease (PAD) characterized by symptoms of intermittent claudication or critical limb ischemia [3]. Diabetic patients are more prone to develop atherosclerosis in comparison to non-diabetic subjects. A variety of mechanisms may contribute to the increased risk of atherosclerosis in patients with T2DM [4]. In addition, chronic condition of arterial disease engenders inflammation and endothelial damage in response to inflammatory markers released in the blood stream of patients with T2DM [5]. Several known risk factors, including hyperglycemia, dyslipidemia, and insulin resistance which characterizes T2DM, acts in concert to target endothelial cells, resulting in oxidative stress and endothelial dysfunction [6]. The combined administration of metformin together with atorvastatin ameliorates inflammation in patients with T2DM [7].

C-reactive protein $(\mathrm{CRP})$ is an inflammatory marker associated with T2DM, obesity, insulin resistance, and cardiovascular disease [8]. Furthermore, according to recent data, CRP is not an acute phase protein only but it is a mediator of atherogenesis, serving as a marker of acute coronary syndromes and cardiovascular disease (CVD) [9].

Notably, it has been recently suggested that common genetic variants within the CRP gene may affect baseline CRP levels and further modulate CRP response in subjects with CVD [10]. CRP gene polymorphisms may also be involved in the development of T2DM by increasing CRP levels [11], while pro-inflammatory genetic profiles are significantly more common in subjects with vascular disease [12]. However, the impact of a specific CRP gene polymorphism such as the $\mathrm{A} 3872 \mathrm{G}$ on the presence and severity of PAD in T2DM remains uncertain.

In the present study, we examined the potential association between Adenine / Guanine (A3872G) CRP gene polymorphism with PAD prevalence in patients with T2DM.

*Corresponding author: Stavroula Papaoikonomou, 14 Lefkosias Street 14123, Likovrisi - Attiki, Greece, Tel: +30 210 2829405, 6932428960; E-mail drstpapaoikonomou@yahoo.gr

Received December 24, 2014; Accepted March 24, 2015; Published March 30 2015

Citation: Papaoikonomou S, Tousoulis D, Tentolouris N, Papageorgiou N, Miliou A, et al. (2015) Genetic Variant of the C-reactive Protein Gene and Prevalence of Peripheral Arterial Disease in Patients with Type 2 Diabetes Mellitus. J Diabetes Metab 6: 529. doi:10.4172/2155-6156.1000529

Copyright: () 2015 Papaoikonomou S, et al. This is an open-access article distributed under the terms of the Creative Commons Attribution License, which permits unrestricted use, distribution, and reproduction in any medium, provided the original author and source are credited. 
Citation: Papaoikonomou S, Tousoulis D, Tentolouris N, Papageorgiou N, Miliou A, et al. (2015) Genetic Variant of the C-reactive Protein Gene and Prevalence of Peripheral Arterial Disease in Patients with Type 2 Diabetes Mellitus. J Diabetes Metab 6: 529. doi:10.4172/21556156.1000529

Page 2 of 5

\section{Material and Methods}

\section{Study population}

The study enrolled 423 Greek Caucasians with T2DM according to the American Diabetes Association criteria [13]. In the total sample the diabetic participants were $\mathrm{n}(\%)$ men: 216 (50.7), with hypertension: 322 (76.3), smokers: 69 (16.6), with PAD: 159 (37.58). Participants were recruited from the registries of Laiko General Hospital and Hippokration Hospital in Athens, based on the inclusion and exclusion criteria. All patients were under standard hypoglycemic, anti-hypertensive, hypolipidemic and anti-platelet therapy.

Presence of PAD was evaluated based on history of revascularization procedures at the leg arteries, typical symptoms of intermittent claudication or clinical signs such as presence of bruits proximally and findings of chronic ischaemia in the periphery such as cold feet, pallor on limb elevation and dependent rubor, trophic skin changes and distal gangrene, ischemic foot ulcers and reduced or non-palpable feet pulses. In addition, clinically significant $(>50 \%)$ stenosis by doppler ultrasonography in lower leg arteries or ankle brachial pressure index $(\mathrm{ABI})<0.9$ we all considered as PAD. Hypercholesterolemia was defined as total cholesterol levels $>200 \mathrm{mg} / \mathrm{dl}$ or the use of lipid lowering agents, according to the definition of the third report of the National Cholesterol Education Program (NCEP) expert panel on detection, evaluation, and treatment of high blood cholesterol in adults (Adult Treatment Panel III) [14]. Subjects were defined as hypertensive patients, if they had medical history of hypertension or arterial blood pressure $\geq 140 / 90 \mathrm{~mm} \mathrm{Hg}$ in two consecutives consultancies or being under antihypertensive medication. Current smokers were defined as those who smoked at least one cigarette per day. Body weight and height were measured in light clothing without shoes and mass index (BMI) was calculated. Moreover, waist and hip circumference were measured and the waist-to-hip ratio (WHR) was calculated. We excluded patients with acute or chronic inflammatory diseases, malignancies, end stage renal disease requiring dialysis, acute coronary syndrome in the last 2 months, and severe liver disease.

The study was approved by the Institutional Ethics Committees, and an informed consent was given by all the participants. Blood samples were taken at the time of recruitment, for genotyping and for evaluation of serum levels of CRP, glucose levels and glycated hemoglobin (HbA1c).

\section{Biochemical measurements}

Venous blood samples were centrifuged at $3,500 \mathrm{rpm}$ at $4^{\circ} \mathrm{C}$ for $15 \mathrm{~min}$, and plasma or serum was collected and stored at $-80^{\circ} \mathrm{C}$ until assayed. Serum concentrations of total cholesterol, high-density lipoprotein cholesterol (HDL-C), low-density lipoprotein cholesterol (LDL-C) were determined by using colorimetric enzymatic method in a Technicon automatic analyzer RA-1000 (Dade-Behring Marburg $\mathrm{GmbH}$, Marburg, Germany). Serum hs-CRP levels were measured by particle-enhanced immunonephelometry (N Latex; Dade-Behring Marburg $\mathrm{GmbH}$ ). $\mathrm{HbAlc}$ was measured by high performance liquid chromatography (ADAMS A1c, ARKRAY, Inc. Kyoto, Japan)

\section{DNA extraction and genotyping}

Genomic DNA was extracted from 2-5 ml of whole blood using standard methods (QIAamp DNA blood kit; Qiagen, West Sussex, UK). For the detection of A3872G (rs1205) polymorphism on CRP gene, we used primer pairs to amplify a part of the gene by Polymerase Chain Reaction (PCR) with the following flanking intronic primers:
rs1205F: 5'cacaagagtggacgtgaa -3' and rs1205R: 5' cttatagacctgggcagt-3'. The resulting product (630bps) was digested by HpyCH4III restriction endonuclease $\left(3 \mathrm{hrs}\right.$ at $37^{\circ} \mathrm{C}$ and resolution by electrophoresis at $1 \%$ agarose gel). Digested fragments were visualized after ethidium bromide staining under ultraviolet light. For PCR quality control, 10\% of the samples were randomly selected and genotyped twice for quality assurance.

\section{Statistical analysis}

Genotype and allele frequencies as well as qualitative variables were compared between groups by chi-square analysis if conformed to Hardy-Weinberg equilibrium proportions and logistic regression analysis was used to estimate odds ratios (OR) and 95\% confidence intervals (CI), of the development of PAD as a function of $\mathrm{A} 3872 \mathrm{G}$ polymorphism. All OR were adjusted for age, gender, hypertension, smoking, duration of diabetes, lipids (HDL-C, LDL-C, triglycerides), $\mathrm{HbAlc}$ and glomerular filtration rate (GFR). Data are shown as mean \pm $\mathrm{SD}, \mathrm{n}(\%)$ or median (interquartile range), as appropriate. Comparisons of continuous variables across the genotypes were performed by unpaired Student's t-test, one-way ANOVA or the non-parametric Mann-Whitney and Kruskal-Wallis tests. All reported P values are based on two-sided tests and compared to a significance level of 5\%. SPSS version 18.0 (SPSS, Chicago, IL) software was used for all the statistical calculations.

\section{Results}

\section{Polymorphisms and sample characteristics}

The demographic and clinical characteristics of the participants stratified by PAD status are shown in Table 1 . Patients with PAD in comparison with those without PAD were older $(\mathrm{P}<0.001)$, had more often hypertension $(\mathrm{P}=0.002)$ and higher arterial systolic blood pressure $(\mathrm{P}<0.001)$, higher WHR $(\mathrm{P}=0.007)$, lower HDL-C $(\mathrm{P}=0.007)$ and lower GFR $(\mathrm{P}<0.001)$. Total cholesterol levels were lower in those with $\mathrm{PAD}$ than in those without $\mathrm{PAD}(\mathrm{P}=0.035)$.

The prevalence of GG, AG and AA polymorphisms of the A3872G was not significantly different between men and women, between smokers and non-smokers and between hypertensive and normotensive participants. Similarly, genotype distribution of log-transformed CRP levels in PAD patients were not significantly different among the three genotypes (GG: $0.61 \pm 0.29$, AG: $0.57 \pm 0.23$, AA: $0.60 \pm 0.23, \mathrm{P}=0.648$ ), (Table 2). Noteworthy, in patients without PAD, GG homozygotes had higher CRP levels compared with carriers of A allele, (GG: $0.61 \pm 0.23$ ) vs. (AG+AA: $0.54 \pm 0.13),(P=0.007)$, (Table 2).

There was a significant gender difference in patients with PAD, (males: GG: $47.4 \%$ vs. AG: $47.8 \%$ vs. AA: $75.0 \%, P=0.014$ ). In the patients with $\mathrm{PAD}$, the GG homozygotes had significantly higher levels of triglycerides; GG: 138.5 (109.75-195.75) mg/dl, in comparison with carriers of "A" allele; AG+AA: 123 (89.50-166.0) mg/dl, (P=0.029), but significantly lower WHR, than the AG, or the AA genotypes, GG: 0.95 (0.9-1.0) vs. AG: 0.96 (0.9-1.0) vs. AA: 1.0 (0.95-1.05), ( $\mathrm{P}=0.031)$. No significant differences were found in serum triglycerides or in WHR in the patients without PAD.

The majority of patients $(n=409,95.1 \%)$ were under standard treatment for diabetes (sulfonylureas, metformin, glitazones, glinides, acarbose, dipeptidyl peptidase 4 inhibitors, glucagon-like peptide-1 receptor agonists or insulin). A total of 317 (73.9\%) were on treatment for hypertension (angiotensin-converting-enzyme inhibitors, angiotensin receptor blockers, diuretics, alpha and beta adrenergic 
Citation: Papaoikonomou S, Tousoulis D, Tentolouris N, Papageorgiou N, Miliou A, et al. (2015) Genetic Variant of the C-reactive Protein Gene and Prevalence of Peripheral Arterial Disease in Patients with Type 2 Diabetes Mellitus. J Diabetes Metab 6: 529. doi:10.4172/21556156.1000529

Page 3 of 5

\begin{tabular}{|c|c|c|c|}
\hline Total & PAD & no PAD & $P$ value \\
\hline CRP A3872G n (\%) & 159(37.58) & $264(62.42)$ & 0.259 \\
\hline Gender (men/women) n (\%) & $86(54.1) / 73(45.9)$ & $130(49.2) / 134(50.8)$ & 0.334 \\
\hline Hypertension (yes /no) n (\%) & $134(84.3) / 25(15.7)$ & $188(71.2) / 76(28.8)$ & 0.002 \\
\hline Smokers (yes/no) n (\%) & $26(16.4) / 133(83.6)$ & $43(16.3) / 221(83.7)$ & 0.986 \\
\hline Age (years) & $69.11 \pm 8.606$ & $65.06 \pm 10.494$ & 0 \\
\hline Duration of diabetes (years) ${ }^{*}$ & $17(9.75-22.0)$ & $10(5.0-18.0)$ & 0 \\
\hline Body mass index $\left(\mathrm{kg} / \mathrm{m}^{2}\right)$ & $28.71 \pm 4.992$ & $29.05 \pm 4.787$ & 0.489 \\
\hline Waist (cm) & $100.92 \pm 11.538$ & $99.24 \pm 12.566$ & 0.172 \\
\hline Waist-to-hip ratio $(\mathrm{m} / \mathrm{m})^{*}$ & $0.96(0.90-1.01)$ & $0.93(0.88-0.99)$ & 0.007 \\
\hline $\mathrm{HbA} 1 \mathrm{c}(\%),(\mathrm{mmol} / \mathrm{mol})$ & $7.47 \pm 1.309,56.56 \pm 9.911$ & $7.30 \pm 1.567,55.27 \pm 11.864$ & 0.251 \\
\hline Glucose (mmol/l) & $8.620 \pm 4.074$ & $8.202 \pm 3.632$ & 0.277 \\
\hline Total cholesterol (mmol/l) & $4.727 \pm 1.047$ & $4.958 \pm 1.110$ & 0.035 \\
\hline LDL cholesterol $(\mathrm{mmol} / \mathrm{l})$ & $2.730 \pm 0.931$ & $2.907 \pm 0.977$ & 0.071 \\
\hline HDL cholesterol (mmol/l) & $1.202 \pm 0.348$ & $1.296 \pm 0.345$ & 0.007 \\
\hline Triglycerides $(\mathrm{mmol} / \mathrm{l})^{*}$ & $1.468(1.084-2.056)$ & $1.435(1.070-1.969)$ & 0.42 \\
\hline hs-CRP $(\mathrm{mg} / \mathrm{ll})^{*}$ & $3.16(3.02-4.1)$ & $3.16(3.02-3.75)$ & 0.589 \\
\hline log hs-CRP & $0.60 \pm 0.26$ & $0.57 \pm 0.19$ & 0.312 \\
\hline GFR (ml/min) & $73.74 \pm 27.88$ & $87.52 \pm 32.648$ & 0 \\
\hline $\mathrm{SBP}(\mathrm{mmHg})$ & $138.96 \pm 17.862$ & $133.06 \pm 14.535$ & 0 \\
\hline $\mathrm{DBP}(\mathrm{mmHg})$ & $76.47 \pm 10.753$ & $77.12 \pm 9.918$ & 0.532 \\
\hline
\end{tabular}

The data are shown as mean \pm SD. *Median values (25-75th percentile)

HbA1c, hemoglobin A1c; HDL cholesterol, high-density lipoprotein cholesterol; LDL cholesterol, low-density lipoprotein cholesterol; GFR, glomerular filtration rate; SBP, systolic blood pressure; DBP, diastolic blood pressure.

Table 1: Clinical and demographic characteristics of subjects with type 2 diabetes mellitus and or no peripheral arterial disease (PAD) by CRP A3872G polymorphism.

\begin{tabular}{|c|c|c|c|c|c|c|c|c|}
\hline \multirow[b]{2}{*}{ CRP A3872G } & \multicolumn{4}{|c|}{ PAD } & \multicolumn{4}{|c|}{ no PAD } \\
\hline & GG & $A G$ & AA & $P$ value & GG & $A G$ & AA & $P$ value \\
\hline hs-CRP $(\mathrm{mg} / \mathrm{l})^{*}$ & $3.16(3.02-5.53)$ & $3.02(3.02-3.45)$ & $3.09(3.02-3.67)$ & 0.207 & $3.16(3.02-4.18)$ & $3.16(3.02-3.45)$ & $3.16(3.02-3.16)$ & 0.481 \\
\hline log hs-CRP & $0.62 \pm 0.29$ & $0.57 \pm 0.24$ & $0.59 \pm 0.24$ & 0.648 & $0.61 \pm 0.24$ & $0.55 \pm 0.14$ & $0.53 \pm 0.13$ & 0.023 \\
\hline CRP A3872G & GG & \multicolumn{2}{|c|}{$A G+A A$} & $P$ value & GG & \multicolumn{2}{|c|}{$A G+A A$} & $P$ value \\
\hline hs-CRP $(\mathrm{mg} / \mathrm{l})^{*}$ & & \multicolumn{2}{|c|}{$3.16(3.02-4.13)$} & 0.083 & & \multicolumn{2}{|c|}{$3.16(3.02-3.16)$} & 0.228 \\
\hline log hs-CRP & & \multicolumn{2}{|c|}{$0.58 \pm 0.24$} & 0.427 & & \multicolumn{2}{|c|}{$0.54 \pm 0.14$} & 0.007 \\
\hline
\end{tabular}

The data are shown as mean \pm SD. *Median values (25-75th percentile).

Hs-CRP; high sensitive C- reactive protein.

Table 2: C-reactive protein levels of subjects with type 2 diabetes mellitus and or no peripheral arterial disease (PAD) by CRP A3872G polymorphism.

receptor antagonists or calcium channel blockers). A total of 226 (52.7\%) were on treatment for dyslipidemia (mainly statins), and 179 (41.5\%) were on anti-platelet therapy (aspirin or clopidogrel). Among the three genotypes no significant differences were found in the use of hypoglycemic (GG vs AG vs AA), (213 (95.9\%) vs $110(94.8 \%)$ vs 83 (93.3\%), $\mathrm{P}=0.606)$, anti-hypertensive $(162(73.3 \%)$ vs $85(73.3 \%)$ vs 68 (76.4\%), $\mathrm{P}=0.838)$, hypolipidemic $(117(52.9 \%)$ vs 59 (50.9\%) vs 48 (53.9\%), $\mathrm{P}=0.898)$, or anti-platelet therapy $(88(39.6 \%)$ vs $52(44.8 \%)$ vs $38(42.7 \%), \mathrm{P}=0.663)$, respectively.

\section{Polymorphisms and PAD prevalence}

Genotype distribution by CRP3872AG polymorphism in subjects with type $2 \mathrm{DM}$ and $\mathrm{PAD}$ are presented in Table 3. Genotype distribution (\%) in PAD patients (GG: 48.1, AG: 29.1, AA: 22.8) was not significantly different compared to non-PAD subjects (GG: 53.8 , AG: 26.7, AA: 19.5), ( $\mathrm{P}=0.258)$. Remarkably, after adjustment for gender, age, duration of diabetes, body mass index (BMI), smoking, hypertension, lipids, HbAlc and GFR, carriers of "A" allele compared with GG homozygotes had increased odds for having PAD; $\mathrm{n} \%$ (AG+AA): 82 (40.0) vs GG: 76 (34.2), ( $\mathrm{P}=0.037)$, (Table 4). In addition, because males with PAD were more often AA homozygotes, we examined the potential effect of the interaction of gender by AA allele on PAD and we did not find significant association in either unadjusted $(\mathrm{P}=0.54)$ or adjusted $(\mathrm{P}=0.62)$ models of logistic regression analysis.

\section{Discussion}

In the present study, we examined the impact of the $A 3872 \mathrm{G}$ polymorphism on CRP gene on the prevalence of PAD in patients with T2DM. Genotype distribution in PAD patients was not significant different compared to non-PAD subjects. The present polymorphism had a significant impact on CRP levels, as they were higher in GG homozygotes compared with carriers of " $\mathrm{A}$ " allele only in patients without PAD. Remarkably, we found a significant difference in the prevalence of PAD, as GG homozygotes showed significantly lower odds for PAD in comparison with carriers of " $\mathrm{A}$ " allele. In the patients with PAD, GG homozygotes had significantly lower WHR than AA homozygotes. This observation may indicate that the fat distribution may play a significant role in the development of PAD, as GG homozygotes who had lower WHR, seem to be protected from the prevalence of PAD.

In a cross-sectional study, involving 3,370 patients with 
Citation: Papaoikonomou S, Tousoulis D, Tentolouris N, Papageorgiou N, Miliou A, et al. (2015) Genetic Variant of the C-reactive Protein Gene and Prevalence of Peripheral Arterial Disease in Patients with Type 2 Diabetes Mellitus. J Diabetes Metab 6: 529. doi:10.4172/21556156.1000529

Page 4 of 5

\begin{tabular}{|c|c|c|c|}
\hline & PAD & no PAD & P value (PAD vs no PAD) \\
\hline Genotype distribution $\mathbf{n}(\%)$ & & & 53.8 \\
\hline GG & 48.1 & 26.7 & \\
\hline AG & 29.1 & 19.5 & 0.258 \\
\hline AA & 22.8 & & \\
\hline Recessive models & & 80.7 & 0.413 \\
\hline GG+AG & 77.4 & 19.3 & \\
\hline AA & 22.6 & 46.2 & 0.256 \\
\hline AG+AA & 51.9 & 53.8 & \\
\hline GG & 48.1 & \\
\hline
\end{tabular}

Table 3: Genotype distribution by CRP A3872G polymorphism in subjects with type 2 diabetes mellitus according to the presence or not of peripheral arterial disease (PAD)

\begin{tabular}{|c|c|c|c|c|c|}
\hline & $X^{2}$ & P value & Unadjusted OR (95\% & CI) & P value \\
\hline $\begin{array}{c}\text { Peripheral arterial } \\
\text { disease }\end{array}$ & & & & \\
\hline AGvsGG & 0.699 & 0.403 & $1.219(0.766-1.941)$ & 0.404 & $1.602(0.934-2.748)$ \\
\hline AAvsGG & 1.078 & 0.299 & $1.310(0.787-2.180)$ & 0.3 & $1.685(0.933-3.041)$ \\
\hline AAvsAG & 0.061 & 0.804 & $1.074(0.610-1.892)$ & 0.804 & $1.029(0.540-1.962)$ \\
\hline AA vs GG+AG & 0.671 & 0.413 & $1.222(0.756-1.977)$ & 0.413 & $1.447(0.837-2.503)$ \\
\hline AG+AA vs GG & 1.289 & 0.256 & $1.257(0.847-1.867)$ & 0.256 & 0.186 \\
\hline
\end{tabular}

*Adjusted parameters: gender, age, duration of diabetes, body mass index, smoking, hypertension, LDL cholesterol, HDL cholesterol, triglycerides, hemoglobin A1c, glomerular filtration rate

OR, odds ratios; $\mathrm{Cl}$, confidence intervals

Table 4: The association between peripheral arterial disease of CRP A3872G polymorphism in subjects with type 2 diabetes mellitus by $\mathrm{x} 2$ and logistic regression analysis.

symptomatic PAD the median values of CRP plasma levels were increased in parallel with the severity of PAD [15]. Other studies reported that the inflammatory biomarkers like fibrinogen and CRP may be involved in the progression of PAD in patients with T2DM patients [16]. Moreover, there was a significant negative correlation between $\mathrm{ABI}$ and high sensitivity CRP (hs-CRP) levels in subjects with T2DM, suggesting an association between serum CRP levels with the severity of PAD [17].

In the present study, we found that the $\mathrm{A} 3872 \mathrm{G}$ polymorphism of the CRP gene has a significant impact on CRP levels only in patients without $\mathrm{PAD}$, as they were higher in GG homozygotes compared to "A" allele carriers. This finding is in accordance with previous data supporting that $\mathrm{A} 3872 \mathrm{G}$ variant in the CRP gene is associated with plasma CRP levels [18]. However, other studies reported that this variant was not associated with CRP levels in healthy elderly subjects [19]. Several other polymorphisms on CRP gene have been proposed as potential modulators of CRP levels and subsequently of CVD risk. According to nested case-control studies, even though common variants of the CRP gene were associated significantly with plasma CRP levels, these associations were not associated with established atherosclerosis [18]. Therefore, the underlying inflammatory processes that are involved in the development and progression of atherosclerosis cannot be explained solely by variation in the CRP gene. Confounders such as duration of diabetes, use of medications with effect on serum CRP levels like statins and angiotensin converting enzyme inhibitors as well as angiotensin receptor blockers and interactions with endothelial function, oxidative stress or other genes controlling inflammatory processes could possibly be involved in the pathogenesis of atherosclerosis.

Visceral fat is infiltrated by macrophages, which trigger insulin resistance, a precursor of T2DM [20] In addition, data derived from a systematic review have shown that waist and hip circumference may improve risk prediction models for CVD and other outcomes [21]. On a random sample of 193 non-insulin-dependent diabetic patients, lower extremity arterial disease was significantly related to central body fat distribution [22]. Our study shows that in patients with PAD, GG homozygotes had significantly lower WHR than AA homozygotes. This observation may indicate that the fat distribution can probably play a significant role in the development of PAD; however this finding requires further confirmation. Recent genome-wide association studies have recognized a great variety of loci associated with BMI and WHR.

Furthermore, several case-controls studies have assessed possible associations between CRP gene variants and clinical vascular disease. The CRP rs1800947, but not the 3872G/A variant, has been suggested as a useful prognostic marker of ischemic stroke [23]. Also, CRP gene -717A allele has been proposed to confer a protective effect against ischemic stroke, while other haplotypes may serve as independent risk markers for ischemic stroke [24]. Remarkably, the present study shows that "A" allele carriage compared with GG homozygosity, conferred an increased risk of PAD, after adjustment for all potential confounders. We also demonstrated for the first time that A3872G polymorphism on CRP gene may have a significant impact on both CRP levels and PAD prevalence in this diabetic population. Indeed, these findings seem fairly consistent, in contrast to the existing literature on CRP gene variants, as aforementioned. Nevertheless, the previous paradoxical findings on the association of CRP gene polymorphisms with CRP levels and vascular disease could be attributed to complex regulatory mechanisms, confounding interactions with conventional risk factors and variable genetic settings of the geographic population of the existing studies. Further studies are required in order to elucidate all controversies in these areas.

Diabetes mellitus is strongly associated with obesity and macrovascular complications including PAD. Inflammatory responses are also associated with T2DM, obesity, insulin resistance, and CVD, while genetic factors may participate in the disease pathogenesis and its clinical manifestations. In the present study we examined the impact of the A3872G polymorphism on CRP gene on the prevalence of PAD in Greek Caucasian patients with T2DM. We found that this variant 
Citation: Papaoikonomou S, Tousoulis D, Tentolouris N, Papageorgiou N, Miliou A, et al. (2015) Genetic Variant of the C-reactive Protein Gene and Prevalence of Peripheral Arterial Disease in Patients with Type 2 Diabetes Mellitus. J Diabetes Metab 6: 529. doi:10.4172/21556156.1000529

Page 5 of 5

may predict CRP levels in this population and may be a marker for increased prevalence of PAD. Moreover, we demonstrated that in patients without PAD, GG homozygotes had significantly lower WHR than AA homozygotes. This observation may indicate that the fat distribution may play a more significant role in the development of PAD, as GG homozygotes who have lower WHR seem to be protected from PAD and explains in part the genetic predisposition for the development of this complication. However, the exact mechanisms by which this inflammatory polymorphism exerts its biological effects are still not clear and further studies perhaps including other inflammatory agents as interleukins, anti-tumor necrosis factor or adiponectin are required to evaluate its contribution to the atherosclerotic pathways of the disease.

\section{Acknowledgement}

To the National University of Athens who supported the research in part by a research grant (S.A.R.G. 70/3/8615)

\section{References}

1. Tousoulis D, Kampoli AM, Papageorgiou N, Papaoikonomou S, Antoniades C et al. (2009) The impact of diabetes mellitus on coronary artery disease: new therapeutic approaches. Curr Pharm Des 15: 2037-2048.

2. de la Hera JM, García-Ruiz JM, Martínez-Camblor $P$, Martín M, Tellería AL, et al. (2013) Real Incidence of Diabetes Mellitus in a Coronary Disease Population. Am J Cardiol 111: 333-338.

3. Joosten MM, Pai JK, Bertoia ML, Rimm EB, Spiegelman D, et al. (2012) Associations between conventional cardiovascular risk factors and risk of peripheral artery disease in men. JAMA 308: 1660-1667.

4. Jude EB, Eleftheriadou I, Tentolouris N (2010) Peripheral arterial disease in diabetes-a review. Diabet Med 27: 4-14

5. Botti C, Maione C, Dogliotti G, Russo P, Signoriello G, et al. (2012) Circulating cytokines present in the serum of peripheral arterial disease patients induce endothelial dysfunction. J Biol Regul Homeost Agents 26: 67-79.

6. Tousoulis D, Kampoli AM, Papageorgiou N, Androulakis E, Antoniades C, et al. (2011) Pathophysiology of atherosclerosis: the role of inflammation. Curr Pharm Des 17: 4089-4110.

7. Tousoulis D, Koniari K, Antoniades C, Papageorgiou N, Miliou A, et al. (2011) Combined effects of atorvastatin and metformin on glucose-induced variations of inflammatory process in patients with diabetes mellitus. Int J Cardiol 149: $46-49$

8. Thorand B, Löwel H, Schneider A, Kolb H, Meisinger C, et al. (2003) C-reactive protein as a predictor for incident diabetes mellitus among middle-aged men: results from the MONICA Augsburg cohort study, 1984-1998. Arch Intern Med 163: 93-99.

9. Tousoulis D, Hatzis G, Papageorgiou N, Androulakis E, Bouras G, et al. (2012) Assessment of acute coronary syndromes: focus on novel biomarkers. Curr Med Chem 19: 2572-2587.
10. Shen J, Arnett DK, Parnell LD, Peacock JM, Lai CQ et al. (2008) Association of common $C$-reactive protein (CRP) gene polymorphisms with baseline plasma CRP levels and fenofibrate response: the GOLDN study. Diabetes Care 31 910-915.

11. Wolford JK, Gruber JD, Ossowski VM, Vozarova B, Antonio Tataranni P, et al. (2003) A C-reactive protein promoter polymorphism is associated with type 2 diabetes mellitus in Pima Indians. Mol Genet Metab 78: 136-144.

12. Flex A, Gaetani E, Angelini F, Sabusco A, Chillà C, et al. (2007) Proinflammatory genetic profiles in subjects with peripheral arterial occlusive disease and critical limb ischemia. J Intern Med 262: 124-130.

13. American Diabetes Association (2013) Diagnosis and classification of diabetes mellitus. Diabetes Care 36 Suppl 1: S67-74.

14. Rodríguez A, Delgado-Cohen H, Reviriego J, Serrano-Ríos M (2010) Risk factors associated with metabolic syndrome in type 2 diabetes mellitus patients according to World Health Organization, Third Report National Cholestero Education Program, and International Diabetes Federation definitions. Diabetes Metab Syndr Obes 4: 1-4.

15. De Haro J, Acin F, Medina FJ, Lopez-Quintana A, March JR (2008) Relationship between the plasma concentration of $\mathrm{C}$-reactive protein and severity of peripheral arterial disease. Clin Med Cardiol 3: 1-7.

16. Bosevski M, Bosevska G, Stojanovska L (2013) Influence of fibrinogen and CRP on progression of peripheral arterial disease in type 2 diabetes: a preliminary report. Cardiovasc Diabetol 12: 29

17. K O T, M S R, G D, S P C, Kumar KMP (2013) A Study of Association of Ankle Brachial Index (ABI) and the Highly Sensitive C - Reactive Protein (hsCRP) in Type 2 Diabetic Patients and in Normal Subjects. J Clin Diagn Res 7: 46-50.

18. Lange LA, Carlson CS, Hindorff LA, Lange EM, Walston J, et al. (2006) Association of polymorphisms in the CRP gene with circulating C-reactive protein levels and cardiovascular events. JAMA 296: 2703-2711.

19. Morita A, Nakayama T, Doba N, Hinohara S, Soma M (2006) Polymorphism of the $\mathrm{C}$-reactive protein (CRP) gene is related to serum CRP Level and arterial pulse wave velocity in healthy elderly Japanese. Hypertens Res 29: 323-331.

20. Attie AD, Scherer PE (2009) Adipocyte metabolism and obesity. J Lipid Res 50: 395-399.

21. Cameron AJ, Magliano DJ, Söderberg S (2013) A systematic review of the impact of including both waist and hip circumference in risk models for cardiovascular diseases, diabetes and mortality. Obes Rev 14: 86-94.

22. Katsilambros NL, Tsapogas PC, Arvanitis MP, Tritos NA, Alexiou ZP, et al. (1996) Risk factors for lower extremity arterial disease in non-insulin-dependent diabetic persons. Diabet Med 13: 243-246.

23. Morita A, Nakayama T, Soma M (2006) Association study between C-reactive protein genes and ischemic stroke in Japanese subjects. Am J Hypertens 19 593-600.

24. Wang Q, Ding H, Tang JR, Zhang L, Xu YJ, et al. (2009) C-reactive protein polymorphisms and genetic susceptibility to ischemic stroke and hemorrhagic stroke in the Chinese Han population. Acta Pharmacol Sin 30: 291-298. 